\title{
Regenerative outlook: offering global solutions for equitable care
}

\author{
Freya Leask*,1 \& Andre Terzic ${ }^{2}$ (D) \\ ${ }^{1}$ Future Science Group, London, N3 1QB, UK \\ ${ }^{2}$ Department of Cardiovascular Medicine; Department of Molecular Pharmacology \& Experimental Therapeutics, Department of \\ Clinical Genomics, Center for Regenerative Medicine, Marriott Heart Disease Research Program, van Cleve Cardiac Regenerative \\ Medicine Program, Mayo Clinic, Rochester, MN 55905, USA \\ *Author for correspondence: f.leask@futuremedicine.com
}

\section{“Regenerative technology could enable us to address the needs of the young and even of those not yet born, for example, in the treatment of congenital conditions."}

First draft submitted: 11 November 2019; Accepted for publication: 11 November 2020; Published online: 27 November 2020

Keywords: evidence-based medicine • global health • healthcare provision • next-generation healthcare - regenerative medicine $\bullet$ regenerative medicine toolkit

When reviewing the last 15 years of regenerative medicine, it is important to think back to the 'why's and 'what's. Answering the question of 'why regenerative medicine' will always come back to assessing the main challenges faced by patients, indeed people. The future prospect of an increasingly aging population and imminent burden of chronic and noncommunicable disease was starting to impact on the individual, population and global society level. Addressing this challenge required more than the treatment of symptoms.

As readers, authors and the editorial team of Regenerative Medicine came together to reflect on 15 years of the journal, this appeared an apropos time to reflect on the field of regenerative medicine: what questions have been answered, what needs have been met, what new questions have appeared and what can we hope for the future of this ever-evolving field?

\section{Reflecting on 15 years of science}

Although the advancing age of the human population can be celebrated as a triumph of humanity, in the early 2000s, the existing solutions on offer by medical science and healthcare were insufficient to address this undoubted challenge [1]. In addition to the demographic shift, there has also been an increasing shift toward diseases and maladies for which we have only mitigating solutions [2]. We had sufficient knowledge to address symptomology but often had little opportunity to progress beyond that.

Therefore, the impetus for the emergence of regenerative medicine as a discipline came largely from two drivers. One driver was an increasing understanding of the intimacy of disease origins which, in turn, pinpointed drug-able targets to take aim for [3]. The second driver came from the development, in recent years, of more disruptive technologies offering ammunition for these targets [4-6].

While we are still at an early stage in which we are addressing the massive challenge of aging and chronic disease, the last 15 years have certainly paved a way toward the regenerative horizon. This provides the opportunity to address not just ageing, but healthy ageing. This paradigm shift in healthcare from care to cure will take society further than simply expanding and extending lifespan; regenerative medicine is poised to match healthspan with lifespan.

\section{Evolution of the regenerative toolkit}

The regenerative medicine toolkit has developed hugely since the early 2000s. In particular, stem cell biology has always been a central pillar of the regenerative toolkit but, over the last 15 years, it has picked up additional components to evolve into a multidisciplinary tool including tissue engineering, functional organoids, exosome 
factories and bioprinted materials, to name a few [7-9]. This now enriched toolbox would not have been recognizable 15 years ago but has been bolstered by scientific innovation at every level.

The field has also seen further advances in material sciences and biotechnology, and notable improvements in techniques in gene editing has seen the addition of another pillar, with gene manipulation becoming an integral part of many new therapeutic solutions [10]. Nevertheless, although the multimodality of regeneration has continued to evolve, the essence of the field in supporting the body's innate ability to heal has remained [11].

An enabling area in which increased development in the field of regenerative medicine has shown to be lacking is readiness of the workforce. Advanced therapies require a proficient workforce with advanced skills and, as with all new fields, development and evolution of the field has uncovered an educational gap which must be bridged via building of substantive curricula [12]. The workforce of the future involves more than future physicians - scientists, engineers, nurses, therapists and paramedical staff; it comprises a polyvalent community which will form an essential contributor to the medicine of tomorrow.

As with all education, the earlier this training starts in the healthcare professional's journey the better. It should follow the form of existing continuing medical education to ensure that professionals have relevant knowledge which has kept up with developments in the field, while ensuring that validated and regulatory authority-approved regenerative therapies are offered as legitimate solutions.

In parallel, as the healthcare profession is being built fit for purpose, equally the public must be educated. The dilemma with any promising area of treatment is offering patients, their families and the public in general true hope, while avoiding giving in to hype, which can be damaging for individuals, as well as the field. The paradigm shift in care modality requires a corresponding shift in mindset, which has not yet been considered and prepared for. Helping all relevant stakeholders understand what validated regenerative options are available should be a key goal of the entire regenerative ecosystem.

Effective collection and communication of evidence, and the stringency of the regulatory process, are required for the ultimately successful delivery of any regenerative medicine treatment [13]. To those peddling hype, this stringency can seem restrictive; however, effective guidelines are an important part of the present and future regenerative medicine gamut. This will ensure the science is successfully brought into the regulatory, qualitycontrolled and compliance-sanctioned space, to form a string in the bow of future evidence-based care [14].

\section{Future opportunities \& remaining obstacles}

It is clear that regenerative medicine is a nascent field that is still rapidly evolving and will continue to have huge ramifications on the healthcare industry. While it is likely that future regenerative treatments will focus on the aging population, the needs of which brought this specialty into life, this will be a true technological revolution. Regenerative technology could enable us to address the needs of the young and even of those not yet born, for example, in the treatment of congenital conditions [15].

The next 15 years is likely to continue the evolution of this technology as it becomes increasingly precise, going after familiar and new targets that are increasingly specific. In turn, this will lead to a lexicon advancement as the field pivots from emphasizing the nature of the technology or mechanism of action, to emphasizing truly regenerative outcomes.

This will have many implications, both in terms of availability of regenerative medicines but also in the building of pathways and processes to enable revolutionary science to benefit patients [16]. Over the last 15 years, confidence in this modality has increased as science is built upon, iterative translational know-how has continued to grow and newly acquired knowledge is applied to new scenarios. Indeed, the experience required for successful regenerative therapy translation, such as how to engineer the supply chain and how to bio-manufacture the regenerative technology, was not yet in existence. However, alongside the experiences of patients and healthcare systems in receiving or delivering regenerative interventions, it has become increasingly vital, which attests to the ongoing maturation of the field and clinical practice uptake.

One challenge that remains is that at the stage of clinical observation and long-term follow-up, the outcomes are mixed. All medical specialties, not just regenerative medicine, must further their understanding of why certain patients respond to treatments favorably and others do not. Therefore, distinguishing responders from nonresponders and, importantly, proactively finding means to adequately then select the patients that have the highest likelihood to respond, will be a major next milestone to be reached in this field [17]. There are many facets to this problem - from identification of suitable outcome markers to modes of therapeutic delivery and delivery timing - and overcoming it will have huge benefits to all areas of medicine and healthcare. 
Here, the concept of regenerative principles diffusing and integrating across medical specialties is paramount; indeed, a natural evolution aimed to embed regenerative science into existing specialties, enriching their respective toolkits and offerings, will broaden its reach. The seamless integration of regenerative medicine will provide medicine with the ability to heal and address the needs of individuals and populations alike, improving outcomes by expanding treatment options, as expansion of outcome-predicting markers targets patients with the greatest potentiality to respond.

Another major challenge lies in the scalability, or lack thereof, of the regenerative product. The long-term success of the field will critically depend on the ability of developers, manufacturers and healthcare providers to deliver homogenous standardized scalable regenerative products. Thus, continued evolution of advanced biomanufacturing and biomanufacturing readiness will be another important pivot as the space moves from concept to reality [18]. However, the final form of this evolution is less certain. Biomanufacturing settling may take place within the healthcare setting as an integral part to the system, or whole new industries may evolve in parallel or adjacent to healthcare systems, adapting to each system's particular need. In the near future, the industry can certainly anticipate concurrent development and testing of many models before a sustainable and effective one, likely a hybrid, is adopted.

A third domain with huge potential for improvement and development is readiness, whether that be biomanufacturing and workforce readiness or supply chain and delivery readiness. In other words, is society in general and healthcare specifically ready for a regenerative model of care? Despite the revolutionary nature of this technology, a successful regenerative model of care will not be disruptive in terms of existing healthcare systems. It will be innovative and is likely to be the driving force behind a push to curative solutions, but it must seamlessly integrate into today's model of care in order for us to realize a model of care suitable for the needs of the world in 2030 and beyond.

\section{Role of the publisher in supporting appropriate dissemination}

Discussion of the importance of evidence generation and communication, and evolving regulation keeping pace with innovation, is incomplete without discussion of the role of the media in the regenerative medicine ecosystem. The peer-reviewed Regenerative Medicine, and the associated eCommunity RegMedNet, have an enduring responsibility as trusted forums to provide space and ongoing opportunities where the professional community but also potential consumers and users of regenerative technology and regenerative therapies can find trusted, timely information, as well as support open sharing of trusted information and diverse experiences.

As any medical discipline moves from science fiction to science fact, another key consideration is access, affordability and healthcare equity; the belief that all should have the opportunity to achieve their full healthcare potential $[19,20]$. These advancements should not be part of daily practice for just the lucky ones, and there is a hugely important socioeconomic imperative to build a system of regenerative care for all. Currently, these early approvals have seen enormous price tags and may be prohibitively expensive. However, in the long run, they may prove more cost-effective by reducing the cost associated with long-term care and treatment. By preventing progression of the disease, we can avoid a highly costly series of irreversible events by interacting and intervening much earlier, and ensuring curative, more definitive, options.

Finally, in order for regenerative medicine to truly benefit the patients who need it most, this must be a global effort not restricted to any particular region. As long as the development of regenerative care comes from unison of forces, recognizing global challenges, it will transform into an offering of a truly global solution.

\section{Acknowledgments}

The authors thank Professor C Mason (UCL and Avrobio) for his invaluable discussion which led to the generation of this manuscript.

\section{Financial \& competing interests disclosure}

A Terzic holds the Marriott Family Professorship in Cardiovascular Diseases Research, and is Michael S and Mary Sue Shannon Director of the Mayo Clinic Center for Regenerative Medicine. A Terzic is supported by the National Institutes of Health (R01 HL134664), Marriott Family Foundation, Van Cleve Cardiac Regenerative Medicine Program, Michael S and Mary Sue Shannon Family, and Center for Regenerative Medicine at Mayo Clinic. A Terzic is co-inventor on regenerative sciences-related intellectual property disclosed to Mayo Clinic. Previously, Mayo Clinic has administered research grants from Celyad. Mayo Clinic and A Terzic have interests in Rion LLC. F Leask is employed by Future Science Group. The authors have no other relevant affiliations or financial 
involvement with any organization or entity with a financial interest in or financial conflict with the subject matter or materials discussed in the manuscript apart from those disclosed.

No writing assistance was utilized in the production of this manuscript.

\section{References}

1. Terzic A, Waldman SA. Chronic diseases: the emerging pandemic. Clin. Transl. Sci. 4(3), 225-226 (2011).

2. Partridge L, Deelen J, Slagboom PE. Facing up to the global challenges of ageing. Nature 561(7721), 45-56 (2018).

3. Wells JM, Watt FM. Diverse mechanisms for endogenous regeneration and repair in mammalian organs. Nature 557(7705), 322-328 (2018).

4. Terzic A, Nelson TJ. Regenerative medicine primer. Mayo Clin. Proc. 88(7), 766-775 (2013).

5. De Luca M, Aiuti A, Cossu G et al. Advances in stem cell research and therapeutic development. Nat. Cell Biol. 21(7), 801-811 (2019).

6. Blau HM, Daley GQ. Stem cells in the treatment of disease. N. Engl. J. Med. 380(18), 1748-1760 (2019).

7. Culme-Seymour EJ, Davie NL, Brindley DA, Edwards-Parton S, Mason C. A decade of cell therapy clinical trials (2000-2010). Regen. Med. 7(4), 455-462 (2012).

8. Kang HW, Lee SJ, Ko IK, Kengla C, Yoo JJ, Atala A. A 3D bioprinting system to produce human-scale tissue constructs with structural integrity. Nat. Biotechnol. 34(3), 312-319 (2016).

9. Behfar A, Terzic A. Regeneration for all: an odyssey in biotherapy. Eur. Heart J. 40(13), 1033-1035 (2019).

10. June CH, Sadelain M. Chimeric antigen receptor therapy. N. Engl. J. Med. 379(1), 64-73 (2018).

11. Mason C. Regenerative medicine 2.0. Regen. Med. 2(1), 11-18 (2007).

12. Wyles SP, Terzic A. Building the regenerative medicine workforce of the future: an educational imperative. Regen. Med. 14(7), 613-615 (2019).

13. Marks PW, Hahn S. Identifying the risks of unproven regenerative medicine therapies. JAMA 324(3), 241-242 (2020).

14. Cossu G, Fears R, Griffin G, Ter Meulen V. Regenerative medicine: challenges and opportunities. Lancet 395(10239), 1746-1747 (2020).

15. Ruano R. Prenatal regenerative fetoscopic interventions for congenital anomalies. BMJ 370, m1624 (2020).

16. Terzic A, Pfenning MA, Gores GJ, Harper CM Jr. Regenerative medicine build-out. Stem Cells Transl. Med. 4(12), 1373-1379 (2015).

17. Yamada S, Arrell DK, Rosenow CS, Bartunek J, Behfar A, Terzic A. Ventricular remodeling in ischemic heart failure stratifies responders to stem cell therapy. Stem Cells Transl. Med. 9(1), 74-79 (2020).

18. Hunsberger J, Harrysson O, Shirwaiker R et al. Manufacturing road map for tissue engineering and regenerative medicine technologies. Stem Cells Transl. Med. 4(2), 130-135 (2015).

19. Gardner J. Distributive justice and regenerative medicine. Regen. Med. 12(7), 865-874 (2017).

20. Webster A. Regenerative medicine and responsible research and innovation: proposals for a responsible acceleration to the clinic. Regen. Med. 12(7), 853-864 (2017). 